

\section{Giant Fibroadenoma in a Middle-Aged Woman Requiring Mastectomy: A Case Report Nwashilli $\mathrm{NJ}^{* 1}$, Obahiagbon I ${ }^{2}$}

1Department of Surgery, ${ }^{2}$ Department of Histopathology, University of Benin Teaching Hospital, Benin City, Edo State, Nigeria

*Correspondence: Dr NJ Nwashilli, Department of Surgery, University of Benin Teaching Hospital, Benin City, Edo State, Nigeria. E-mail: namoforever@yahoo.com; ORCID - https://orcid.org/0000-0003-2714-8024.

Summary

Fibroadenomas are benign tumours of the breast. They are usually single, firm, rubbery masses, slow-growing and well encapsulated. Giant fibroadenomas are fibroadenomas at least $5 \mathrm{~cm}$ in size or at least $500 \mathrm{~g}$ in weight. The peculiarities of the index case include the massive size and weight of the breast, causing asymmetry and tissue distortion with little or no normal breast tissue on ultrasound scan. Also, such massive weight has not been reported in the literature as suggested by extensive search on databases such as Pubmed and Google Scholar. The main concern of the patient was the rapid growth over a year, with the attendant risk of malignancy. The mass was firm, lobulated, with a solitary axillary lymph node. An initial clinical diagnosis of phyllodes tumour was made. However, preoperative Tru-cut biopsy histology suggested fibroadenoma and was confirmed using the excised post-operative specimen. Simple mastectomy with axillary lymph node excision was carried out. In conclusion, a large breast tumour may not be malignant. However, mastectomy may be a treatment option despite the benign nature of the tumour.

Keywords: Axillary lymphadenopathy, Breast tumour, Giant fibroadenoma, Mastectomy, Phyllodes tumour.

\section{Introduction}

Fibroadenomas are benign breast tumours of women which occur between the second and third decades of life, with a prevalence of $2.2 \%$. [1] They are usually single masses, firm in consistency, well encapsulated and slowgrowing. Fibroadenoma occurs as a result of increased oestrogen stimulus and receptor sensitivity and decrease in oestrogen antagonist levels during puberty. [2] Fibroadenoma is described as a giant when it is larger than $5 \mathrm{~cm}$ in size, weighs more than $500 \mathrm{~g}$, or replaces at least $80 \%$ of the normal breast tissue. [3]

It can pose some challenges for the affected woman and the attending physician. These include breast asymmetry/deformity, a cosmetic problem leading to psychological disturbances, difficulty in differentiating it from phyllodes tumour on fine needle biopsy and the appropriate surgical intervention. All these challenges may lead to delay in treatment. Surgical treatment of giant fibroadenomas ranges from simple excision to reduction mammoplasty 
or mastectomy with breast reconstruction. ${ }^{[4]}$ This report is about a middle-aged woman who presented with histologically confirmed giant fibroadenoma and which required mastectomy due to its massive size and weight as well as an unfavourable ratio of diseased breast tissue to the healthy breast tissue. The uniqueness of this case is the massive size which has not been previously reported in the literature.

\section{Case Description}

A 40-year old woman presented with a huge right breast mass of two-year duration. The breast mass was initially small but gradually increased in size. It was painless, with no nipple discharge or retraction and no skin changes. There was no mass in the contralateral breast. The woman had attained menarche at 15 years and her parity was four, (all alive). There was no history of breast disease or malignancy in the family. The rapid growth of the breast mass over one year, with the attendant risk of malignancy, were the immediate concerns of the woman. She had no previous treatment or surgical interventions for the breast mass.
General examination revealed a healthy-looking woman, not pale, afebrile, anicteric, not dehydrated, with no pedal oedema and no significant peripheral lymphadenopathy. The right breast showed a huge mass occupying the entire breast; it was sagging, with a lobulated surface but with no nipple retraction, tenderness, or differential warmth. The mass measured $15 \times 20 \mathrm{~cm}$, and it was freely mobile, unattached to the skin or underlying structure with a $2 \mathrm{~cm}$ solitary mobile axillary lymph node (Figures 1 and 2). The left breast was normal.

The haematocrit was 31\%, (Haemoglobin concentration $10 \mathrm{~g} / \mathrm{dl}$ ), the total white blood cell count was $5 \times 10^{3} / \mu \mathrm{L}$ and the platelet count was $139 \times 10^{3} / \mu \mathrm{L}$. The serum electrolytes, urea and creatinine, random blood sugar, urinalysis, chest $\mathrm{X}$-Ray and electrocardiography showed normal findings. Mammography suggested a right breast mass with malignant features (BI-RADS Category 4) while ultrasonography was suggestive of phyllodes tumour with residual breast tissue. A Tru-cut biopsy for histology confirmed the diagnosis of fibroadenoma with no evidence of malignancy. The histology of the isolated axillary lymph node only showed inflammatory features without malignant changes.

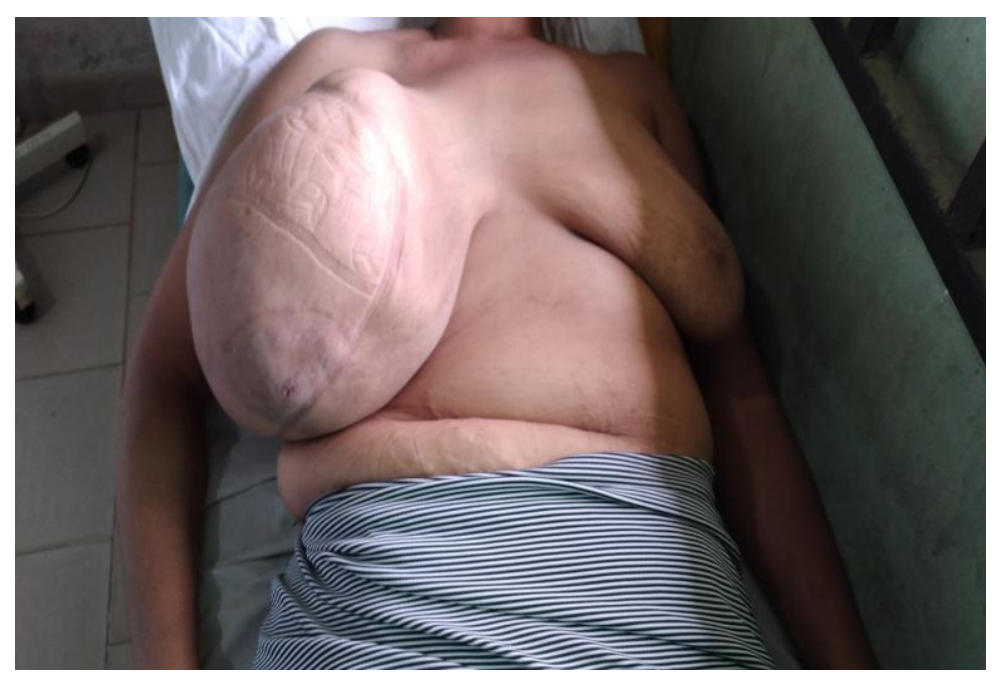

Figure 1: Showing marked breast asymmetry with a huge right breast mass and its nipple at the level of the umbilicus at presentation. 


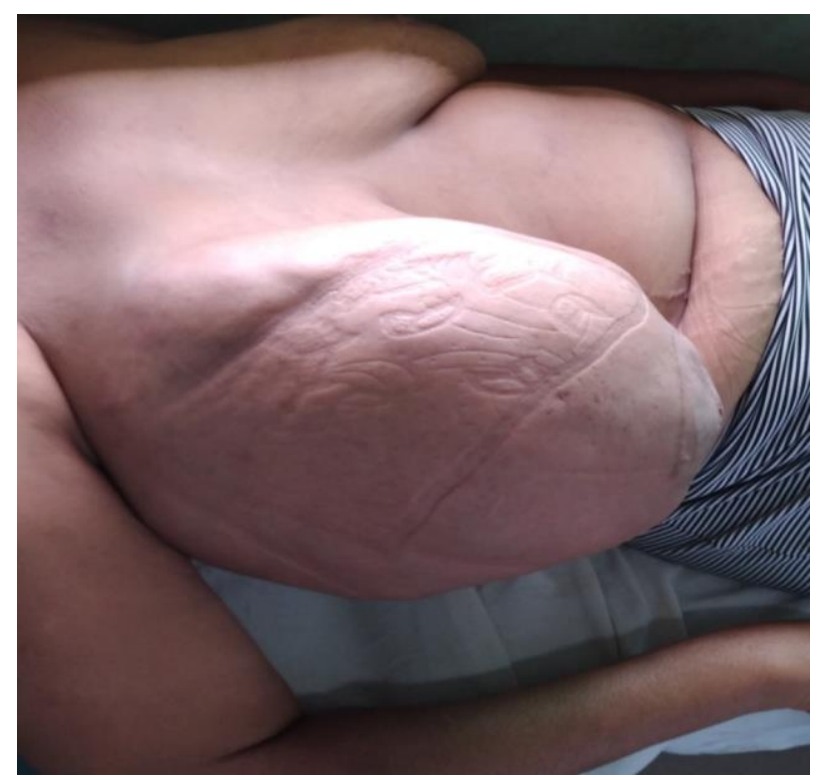

Figure 2: Lateral view of the Breast mass at presentation.

The woman was counselled for simple mastectomy and breast reconstruction using a latissimus dorsi or Transverse Rectus Abdominomyocutaneous (TRAM) Flap. This was aimed at achieving a sufficient breast mound and symmetry with the contralateral breast. She had a simple mastectomy with the removal of the enlarged axillary lymph node but financial constraint precluded breast reconstruction. The choice of simple mastectomy was based on the massive nature of the tumour involving the entire breast with little or no breast tissue as demonstrated on ultrasound. The operative specimen weighed $6.8 \mathrm{~kg}$ (Figure 3 ). The removed tumour was re-confirmed as fibroadenoma without any evidence of malignancy (Figure 4). The mastectomy wound healed well with a good outcome. The woman is currently being followed up at the out-patient clinic with no evidence of local recurrence.
Giant (Juvenile/Adult) fibroadenoma is a rare variant of fibroadenoma characterized by large size and rapid growth. ${ }^{[5]}$ It is most commonly seen in females of Afro-Caribbean or East Asian descent with bimodal age distribution and thus, occurs in adolescent and premenopausal women. [6] The index case occurred in a middle-aged, Nigerian woman.

Varying weights of excised giant fibroadenoma have been reported in databases such as the Pubmed and Google scholar but there is no report of such large weight tumour of $6.8 \mathrm{~kg}$ like in this index case. The largest adult giant fibroadenoma size reported in the literature measured $23 \times 28$ $\mathrm{cm}$, but the weight was not stated. [7] Nevertheless, the tumour mass in the index case measured $15 \times 20 \mathrm{~cm}$. Tumours of smaller sizes and weights have also been reported. The various sizes and weights may determine the type of surgery required.

\section{Discussion}




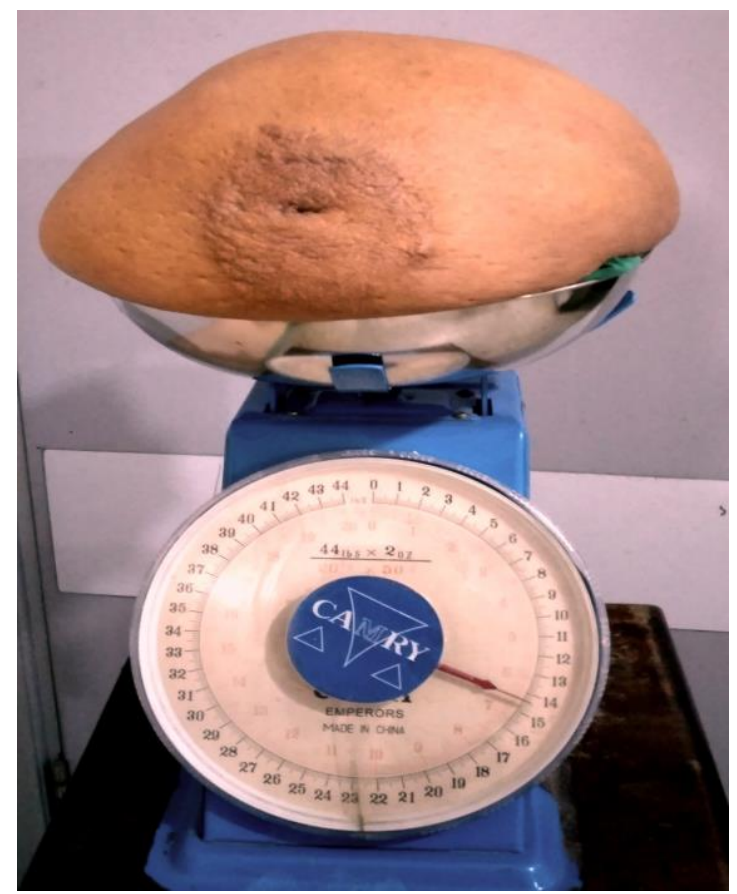

Figure 3: Operative breast tissue specimen weighing $6.8 \mathrm{~kg}$ immediately after surgery

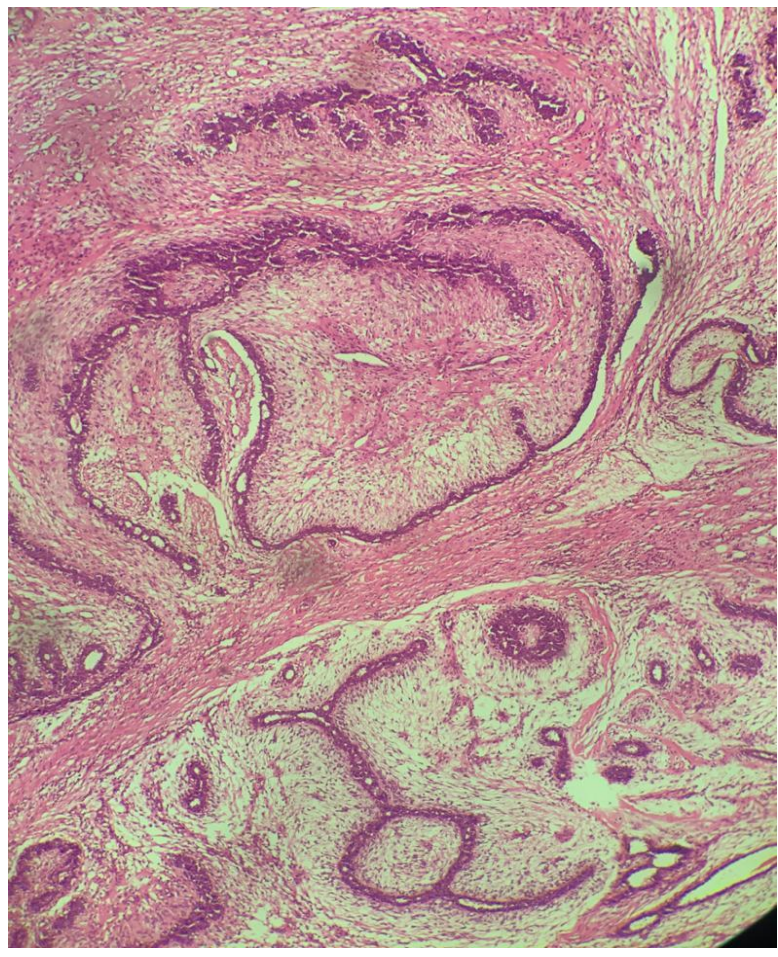

Figure 4: Histopathological sections of the breast tissue showing a benign neoplasm with ducts and acini lined by bland epithelial and myoepithelial cells and compressed into slit-like, often anastomosing channels by proliferating fibromyxoid stroma. 
Breast reconstruction is not commonly performed after mastectomy for giant fibroadenoma but may be required in some cases. In a systematic review with diagnostic and treatment recommendations for giant juvenile fibroadenoma, breast reconstruction was carried out in $27(17.6 \%)$ of 153 cases. [8] The index case did not have breast reconstruction due to financial constraint, even though she desires to have delayed reconstruction in the nearby future.

The surgical treatment of giant fibroadenoma is debatable in the literature. Surgical options vary from simple enucleation to mastectomy with or without immediate or delayed breast reconstruction. Achebe and colleagues in their study of 27 patients with severe breast asymmetry caused by giant fibroadenoma, with an average size of $15 \mathrm{~cm}$ and weight of $1.5 \mathrm{~kg}$ at the University of Nigeria Teaching Hospital, Enugu, Enugu State, southeast Nigeria, carried out reduction mammoplasty with good cosmetic outlook. [9] Their good outlook was achieved by considering a difference in the position of the nipple-areola complex of $6 \mathrm{~cm}$ as significant breast asymmetry to be a criterion for reduction mammoplasty. Mastectomy was carried out in the index case due to the large size $(15 \times 20 \mathrm{~cm})$, the massive weight of $6.8 \mathrm{~kg}$, unfavourable ratio of diseased breast tissue to the normal breast tissue on ultrasound scan, increased age (middle age) associated with the risk of malignancy, breast distortion, and deformity. Dolmans, et al. similarly carried out mastectomy in a patient with a giant fibroadenoma $(9 \mathrm{~cm}$ size), but their patient also had breast reconstruction which was not performed in the index case because she could not afford it. [4] The indication for mastectomy in the series of Dolmans, et al. was the unfavourable ratio of diseased breast tissue to healthy tissue.

A good differential diagnosis of a giant fibroadenoma is benign/borderline phyllodes tumour. The features of both may overlap, making it difficult to distinguish on fine-needle aspiration and cytology (FNAC), but core needle biopsy for histopathology makes the distinction. [10] Other possible lesions in this 40-year old woman may include giant lipoma, large breast abscess, large cysts of fibrocystic disease, parasitic cysts and breast cancer. More importantly, breast cancer needs to be excluded in a middle-aged woman with such a massive breast lesion.

Though the initial diagnosis was phyllodes tumour, based on the clinical appearance of the mass, a Tru-cut biopsy specimen confirmed the lesion as fibroadenoma on histology. Other diagnostic modalities used by pathologists to distinguish phyllodes tumour from fibroadenoma include immunochemistry and molecular/cytogenetic studies. Unfortunately, molecular/cytogenetic studies are currently unavailable in resource-limited settings like ours. On immunohistochemistry, benign phyllodes tumour and fibroadenoma have many immunohistochemical markers in common, hence immunohistochemistry may not help distinguish between the two lesions in practice. [11] Ki67 labelling may be higher in benign phyllodes tumour than in fibroadenoma but many cases of benign phyllodes will have a similar proliferative index to a fibroadenoma. [11] Ki67 becomes more useful as one moves towards borderline and malignant phyllodes tumour where the mitotic count is higher. [11] Neither immunohistochemistry nor molecular/cytogenetic study was carried out in the index case.

A conference of pathologists had an extensive review of the histology slide vis-a-viz the gross and microscopic features. Grossly, phyllodes tumours tend to be larger and not circumscribed (unencapsulated), unlike fibroadenoma that is encapsulated. Occasionally, fibroadenomas can 
achieve giant size. On Haematoxylin \& Eosin $(\mathrm{H}$ \& E) staining for microscopy, the fibroadenoma is a proliferation of both ducts/acini and intralobular fibromyxoid stroma, whereas phyllodes tumour is a stromal overgrowth only, sparing the epithelium and imparting a leaf-like architecture to the tumour. [12] Also, there is increased periductal cellularity in phyllodes tumour, which is absent in fibroadenoma. [12] Borderline and malignant phyllodes tumours are not usually confused with fibroadenoma which is usually benign. In borderline and malignant phyllodes tumour, there is hypercellularity, increased nuclear pleomorphism and mitotic index. [12] Based on these observations, the pathologists concluded the tissue obtained from the mass was a fibroadenoma. After excision of the tumour, it was thoroughly reviewed and a final diagnosis of fibroadenoma was further reconfirmed in the absence of features of malignancy.

The message in this case report is that a huge, fast-growing tumour of the breast may not necessarily be malignant. There is a need to have a thorough review of histopathological slides, preferably by a team of histopathologists and correlate it with clinical features when diagnostic dilemma exists. Mastectomy may be a treatment option despite the diagnosis being benign.

\section{Conclusion}

Giant fibroadenoma of the breast is a rare tumour which occurs in younger females but may occur in middle-aged females causing diagnostic dilemma as the risk of malignancy increases at that age. Thorough clinical, radiological and histopathological evaluations are necessary to ensure an accurate diagnosis.

Authors' Contributions: NNJ conceived and designed the study, did data collation and drafted the manuscript. OI participated in data collection and interpretation, and histopathological diagnosis. Both authors approved the final version of the manuscript.

Conflict of Interest: None declared.

Funding: Self-funded.

Publication History: Submitted 24 July 2020; Accepted 07 October 2020.

\section{References}

1. Santen RJ, Mansel R. Benign breast disorders. $N$ Engl J Med 2005; 353: 275-285. doi:10.1056/NEJMra035692.

2. Merchant DJ. Benign breast disease. Obstet Gynecol Clin North Am 2002; 29: 1-20. doi:10.1016/s0889-8545(03)00048-2.

3. Jayasinghe $Y$, Simmons PS. Fibroadenoma in adolescence. Curr Opinion Obstet Gynecol 2009; 21: 402-406. doi:10.1097/GCO.0b013e32832fa06b.

4. Dolmans GH, Hoogbergen MM, van Rappard JH. Giant fibroadenoma of one breast: immediate bilateral reconstruction. J Plast Reconstr Aesthet Surg 2007; 60: 1156-1157. doi:10.1016/j.bjps.2007.01.005.

5. Arowolo OA, Akinkuolie AA, Adisa AO, Obonna GC, Olasode BJ. Giant fibroadenoma presenting like fungating breast cancer in a Nigerian teenager. Afr Health Sci 2013; 13: 162-165. doi:10.4314/ahs.v13i1.23.

6. Hanna RM, Ashebu SD. Giant fibroadenoma of the breast in an Arab population. Australas Radiol 2002; 46: 252-256. doi:10.1046/j.14401673.2002.01054.x.

7. McCague A, Davis JV. Giant fibroadenoma in a 22-year old patient: case report and literature review. Breast Dis 2010; 31: 49-52. doi:10.3233/BD-2009-0288.

8. Sosin M, Pulcrano M, Feldman ED, Patel KM, Nahabedian MY, Weissler MJ, et al. Giant juvenile fibroadenoma: a systematic review with diagnostic and treatment 
recommendations. Gland Surg 2015; 4: 312321. doi:10.3978/j.issn.2227-684X.2015.06.04.

9. Achebe JU, Njeze GE, Okwesili OR. Treatment of unilateral giant fibroadenoma by breast reduction skin incision: the inverted " $T$ " technique. Niger J Clin Pract 2014; 17: 4346. doi:10.4103/1119-3077.122835.

10. Bandyopadhyay R, Nag D, Modal SK, Mukhopadhyay S, Roy S, Sinha SK. Distinction of phyllodes tumour from fibroadenoma: Cytologist's perspective. J Cytol 2010; 27: 59-62. doi:10.4103/09709371.70739 .
11. Yohe S, Yeh IT. "Missed" diagnoses of Phyllodes tumour on breast biopsy: pathological clues to its recognition. Int J Surg Pathol 2008; 2: 137-142. doi:10.1177/10668969.07311378.

12. Jacobs TW, Chen YY, Guinee Jr DG, Holden JA, Cha I, Bauermeister DE, et al. Fibroepithelial lesions with cellular stroma on breast core needle biopsy: Are there predictors of outcome on surgical excision? Am J Clin Pathol 2005; 124: 342-354. doi:10.1309/5N2C-4N5X-CBBX-W8JL. 